\title{
The alternative splicing factor PSI regulates P-element third intron splicing in vivo
}

\author{
Melissa D. Adams, Rosa S. Tarng, ${ }^{1}$ and Donald C. Rio ${ }^{2}$ \\ Department of Molecular and Cell Biology, University of California, Berkeley, Berkeley, California 94720-3204 USA
}

\begin{abstract}
Splicing of the Drosophila P-element third intron (IVS3) is inhibited in somatic cells, restricting transposase expression to the germ line. Somatic inhibition of IVS3 splicing involves the assembly of a multiprotein complex on a regulatory sequence in the IVS3 $5^{\prime}$ exon. The P-element somatic inhibitor protein (PSI) is a component of this ribonucleoprotein complex and is required for inhibition of IVS3 splicing in vitro. The soma-specific expression pattern of PSI suggests that its low abundance in the germ line allows IVS3 splicing. We demonstrate that ectopic expression of PSI in the female germ line is sufficient to repress splicing of an IVS3 reporter transgene. We also show that IVS3 splicing is activated in somatic embryonic cells in the presence of an antisense PSI ribozyme. These results support the model that PSI is a tissue-specific regulator of IVS3 splicing in vivo.
\end{abstract}

[Key Words: Pre-mRNA splicing; alternative splicing regulation; RNA-binding protein]

Received September 18, 1996; revised version accepted November 7, 1996.

RNA splicing is the process by which intervening sequences, introns, are excised from primary transcripts to generate functional eukaryotic messenger RNAs. Splicing occurs via a two-step trans-esterification reaction catalyzed by a multicomponent ribonucleoprotein complex called the spliceosome. The spliceosome is composed of the U1, U2, U5, and U4/U6 small nuclear ribonucleoprotein particles (snRNPs) and many non-snRNP spliceosome-associated proteins that are assembled onto the precursor mRNA (pre-mRNA) in a stepwise manner (Moore et al. 1993).

Alternative pathways of pre-mRNA splicing are often utilized by the cell to regulate gene expression in a temporal or cell type-specific manner or to direct the synthesis of structurally related protein isoforms with different functions (Green 1991; Rio 1993). Biochemical and genetic studies of a variety of alternative splicing events have led to the identification of splicing regulatory factors that act in a tissue-, stage-, or sex-specific manner. Biochemical studies using model introns indicate that these alternative splicing factors are often found in complexes with general non-snRNP splicing factors assembled on regulatory sequences in the premRNA. These RNP complexes modulate snRNP-premRNA interactions to influence splice site selection either positively, by activating use of an alternative splice

\footnotetext{
${ }^{1}$ Present address: Department of Molecular and Cellular Biology, Harvard University, Cambridge, Massachusetts 02138 USA.

${ }^{2}$ Corresponding author.

E-MAIL don_rio@mendel.berkeley.edu; FAX (510) 642-6062.
}

site, or negatively, by inhibiting selection of a constitutively active site (Adams et al. 1996).

In Drosophila, the tissue-specificity of P-element transposition is regulated at the level of pre-mRNA splicing (Laski et al. 1986). In germ-line cells, all three introns of the P-element transcript are spliced accurately and the resulting mRNA encodes transposase. In somatic cells (as well as in the germ line), the third intron (IVS3) is retained, and, as a result, a truncated protein that represses transposition is expressed (Misra and Rio 1990). Genetic and biochemical studies have revealed that regulation of this tissue-specific splicing event involves inhibition of IVS3 excision in the soma (Laski and Rubin 1989; Siebel and Rio 1990; Chain et al. 1991). Somatic inhibition requires a discrete regulatory sequence in the $5^{\prime}$ exon RNA adjacent to the IVS3 $5^{\prime}$ splice site (Chain et al. 1991; Siebel et al. 1992). In Drosophila somatic cell nuclear extracts, a multiprotein complex forms on this exonic element and prevents U1 snRNP from binding to the accurate IVS3 $5^{\prime}$ splice site, an early step in splice site selection and spliceosome assembly. U1 snRNP binds instead to one of two pseudo-5' splice sites within the upstream exon regulatory element (Siebel et al. 1992). Two components of the multiprotein inhibitory complex have been identified. One of these, hrp48, was originally isolated as a Drosophila hnRNP protein (Matunis et al. 1992a,b). Hrp48 is expressed in both the soma and the germ line and binds to the second pseudo-5' splice site within the regulatory sequence (Siebel et al. 1994, 1995). Inhibition of IVS3 splicing is partially relieved in hrp48 mutant flies consistent with a 
role in somatic repression (L. Hammond, D. Rudner, R. Kanaar, and D. Rio, in prep.). The P-element somatic inhibitor protein (PSI) is also a component of the inhibitory complex and is highly expressed only in the soma (Siebel et al. 1995; J. Warsowe and D.C. Rio, unpubl.). PSI binds specifically to the regulatory RNA sequence and is required for inhibition of IVS3 splicing in somatic extracts (Siebel et al. 1994, 1995).

The finding that PSI is expressed at high levels only in somatic cells suggests that it may be a tissue-specific negative regulator of IVS3 splicing. The reduced level of PSI expression in the Drosophila germ line may prevent efficient formation of the inhibitory complex and allow IVS3 splicing and transposase expression. Here we have tested this model using a maternally expressed promoter to drive expression of a PSI transgene in germ-line cells during oogenesis. We have demonstrated that ectopic expression of PSI in the female germ line is sufficient to repress splicing of an IVS3-containing reporter RNA. Additionally, using a heat-inducible ribozyme targeted against the PSI mRNA, we have shown that reduction of PSI expression in somatic embryonic cells activates somatic splicing of an IVS3-containing reporter transgene. These results establish that PSI, a biochemically identified splicing factor, functions as a regulator of tissuespecific IVS3 splicing in vivo.

\section{Results}

Ectopic expression of a PSI cDNA represses P-element IVS3 splicing in the germ line

To determine whether germ-line expression of PSI is sufficient to inhibit IVS3 splicing, we generated transgenic strains of flies that ectopically express the PSI cDNA in the Drosophila ovary under transcriptional control of the hsp83 promoter (Fig. 1A). This promoter is active in ovarian germ-line nurse cells (Ding et al. 1993). Of five independent transformant lines recovered, only one, line 29-1, exhibited normal levels of viability and female fertility. The other four lines displayed reduced viability when compared with the parental $\mathrm{w}^{1118}$ strain, could not be maintained as homozygous stocks, and ultimately died. A mobilization screen for additional transgene insertions was initiated before the original stocks were lost, and three new independent transformants were recovered. Two of these transformants died before they could be mated, but one, line 103-1, was healthy and homozygous viable. The high proportion of transformant lines displaying reduced viability suggests that ectopic overexpression of PSI has deleterious effects. These effects could be a result of ectopic germ-line expression of PSI or overexpression of PSI in somatic cells where the hsp83 promoter is also active (Ding et al. 1993). However, we cannot rule out the possibility that these phenotypic effects were attributable to mutations introduced during P-element insertion.

Whole mount immunostaining and immunoblotting of ovaries and ovary nuclear extracts from the 29-1 and 103-1 transformants with anti-PSI antibodies did not re-
A

Transgene for ectopic germline expression

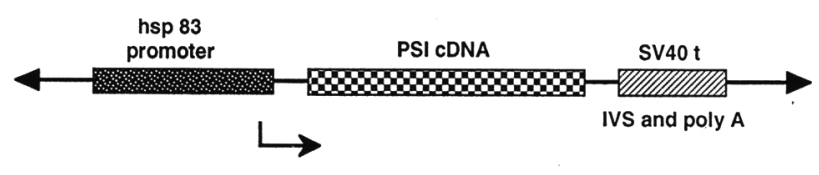

Reporter transgene for IVS3 splicing

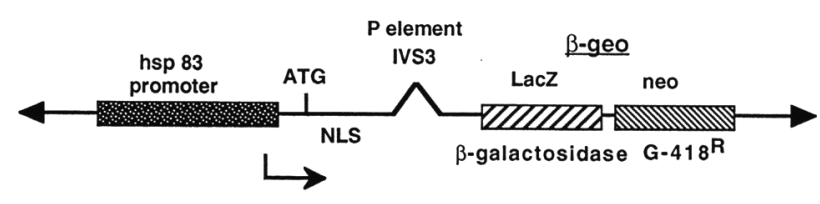

B

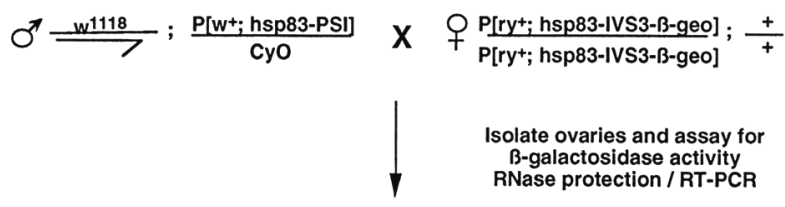

$\nmid \frac{\text { P[ry+; hsp83-IVS3-ß3-geo] }}{w^{1118}} ; \frac{\mathrm{P}\left[\mathrm{w}^{+} ; \text {hsp83-PSI] }\right.}{+} \quad \begin{gathered}\text { Experimental class } \\ \text { PSI and reporter }\end{gathered}$

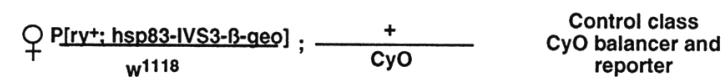

Figure 1. Ectopic PSI and IVS3 reporter transgenes and genetic assay for IVS3 splicing. (A) Schematic diagram of the transgene used to express PSI in germ-line ovarian cells and the $\mathrm{P}\left[\mathrm{ry}^{+}\right.$; hsp83-IVS3- $\beta$ geo] reporter transgene (Roche et al. 1995) used to assay germ-line IVS3 splicing. Expression of both transgenes is driven by the hsp83 promoter, which is active during oogenesis (Ding et al. 1993). (B) The genetic cross used to assay the effect of ectopic PSI expression on IVS3 splicing. Males heterozygous for the $\mathrm{P}\left[\mathrm{w}^{+}\right.$; hsp83-PSI $]$transgene and the dominantly marked CyO balancer were crossed to virgin females homozygous for the $\mathrm{P}\left[\mathrm{ry}^{+}\right.$; hsp83-IVS3- $\beta$ geo] reporter. Ovaries from experimental ( $\mathrm{P}\left[\mathrm{ry}^{+}\right.$; hsp83-IVS3- $\beta$ geo $] ; \mathrm{P}^{2} \mathrm{w}^{+}$; hsp83-PSI]) female progeny and their control $\left(\mathrm{P}\left[\mathrm{ry}^{+}\right.\right.$; hsp83-IVS3- $\beta$ geo]; $\left.\mathrm{CyO}\right)$ sisters were isolated and assayed fro IVS3- $\beta$ geo splicing.

veal a significant increase in the low level of PSI protein in this tissue when compared with the $\mathrm{w}^{1118}$ control. However, the chimeric hsp83-PSI transcript could be detected in the ovary RNA from these flies /data not shown). This observation may explain why these particular transformants do not exhibit any of the adverse biological effects observed for the other independently isolated transformants described above.

To study the effect of ectopic PSI expression on germline IVS3 splicing, males heterozygous for the $\mathrm{P}\left[\mathrm{w}^{+}\right.$; hsp83-PSI] transgene were crossed to virgin females homozygous for an IVS3-containing splicing reporter (Fig. 1). The reporter transgene contains a fusion of the P- 
element third intron, including the upstream exonic regulatory sequence, to the lac $Z$ gene under control of the hsp83 promoter $\left(\mathrm{P}\left[\mathrm{ry}^{+}\right.\right.$; hsp83-IVS3-Bgeo]; Roche et al. 1995). Expression of $\beta$-galactosidase from this construct requires the accurate removal of IVS3 and has been shown to be restricted to the germ-line ovarian cells (Roche et al. 1995). Two classes of female progeny were obtained from this cross: a control class containing one copy of the reporter transgene and an experimental class with one copy of the PSI transgene and one copy of the reporter (Fig. 1B). Ovaries were isolated from females of each class and assayed for $\beta$-galactosidase activity using a histochemical staining assay.

Ectopic PSI expression in the ovary, from the 29-1 transgene, resulted in a decrease in the level of germ-line $\beta$-galactosidase activity (Fig. 2A-C), indicating a reduction in the level of IVS3 splicing. The magnitude of the decrease in staining varied among experimental ovaries from a single cross (Fig. 2, cf. B and C). Additionally, in the experimental ovarioles with both high and low levels of staining, we noted that there was a consistent mosaic pattern of staining among the nurse cell nuclei, as if PSI was not efficiently expressed from the transgene at the same time or level in all cells. Uniform staining of the nurse cell nuclei was consistently observed in the control ovarioles. It is noteworthy that line 29-1 exhibits mosaicism for the $\mathrm{w}^{+}$eye color marker present on the PSI transgene. This is consistent with the P element being inserted at a genomic locus that is subject to position-effect variegation (Hazelrigg et al. 1984). Variegated PSI expression in the ovary could account for the mosaic pattern of inhibition observed in the staining assay. In a similar experiment, transformant 103-1 gave a modest reduction in the level of $\beta$-galactosidase activity, but did not exhibit a mosaic pattern of nurse cell staining as did line 29-1 (data not shown). This observation is consistent with variegated expression of PSI from the 29-1 transgene causing the mosaic pattern of staining repression.

The reduction in $\beta$-galactosidase staining was a result of repression of IVS3 splicing, because expression of a reporter transgene from which IVS3 has been precisely

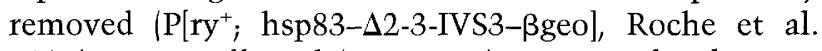
1995) was unaffected (Fig. 2D,E). Staining levels were similar in ovarioles from both the control and the experimental classes, and there was no mosaic pattern of nurse cell staining. $\beta$-Galactosidase expression from the $\Delta 2-3$ reporter transgene was also observed in the somatically derived follicle cells surrounding the developing oocyte, because the requirement for IVS3 splicing was removed.

To analyze directly the effect of ectopic PSI expression on IVS3 reporter splicing, both ribonuclease (RNase) protection and quantitative reverse transcription-polymerase chain reaction (RT-PCR) assays were performed on ovary RNA from both the control and the experimental females. In the RNase protection assay, a 231 -nucleotide probe fragment was protected from digestion by spliced IVS3 reporter RNA and a 461-nucleotide fragment was protected by the unspliced RNA (Fig. 3A). A probe for $\alpha$-tubulin mRNA was included in the reactions as a standard for the amount of target RNA in each sam-

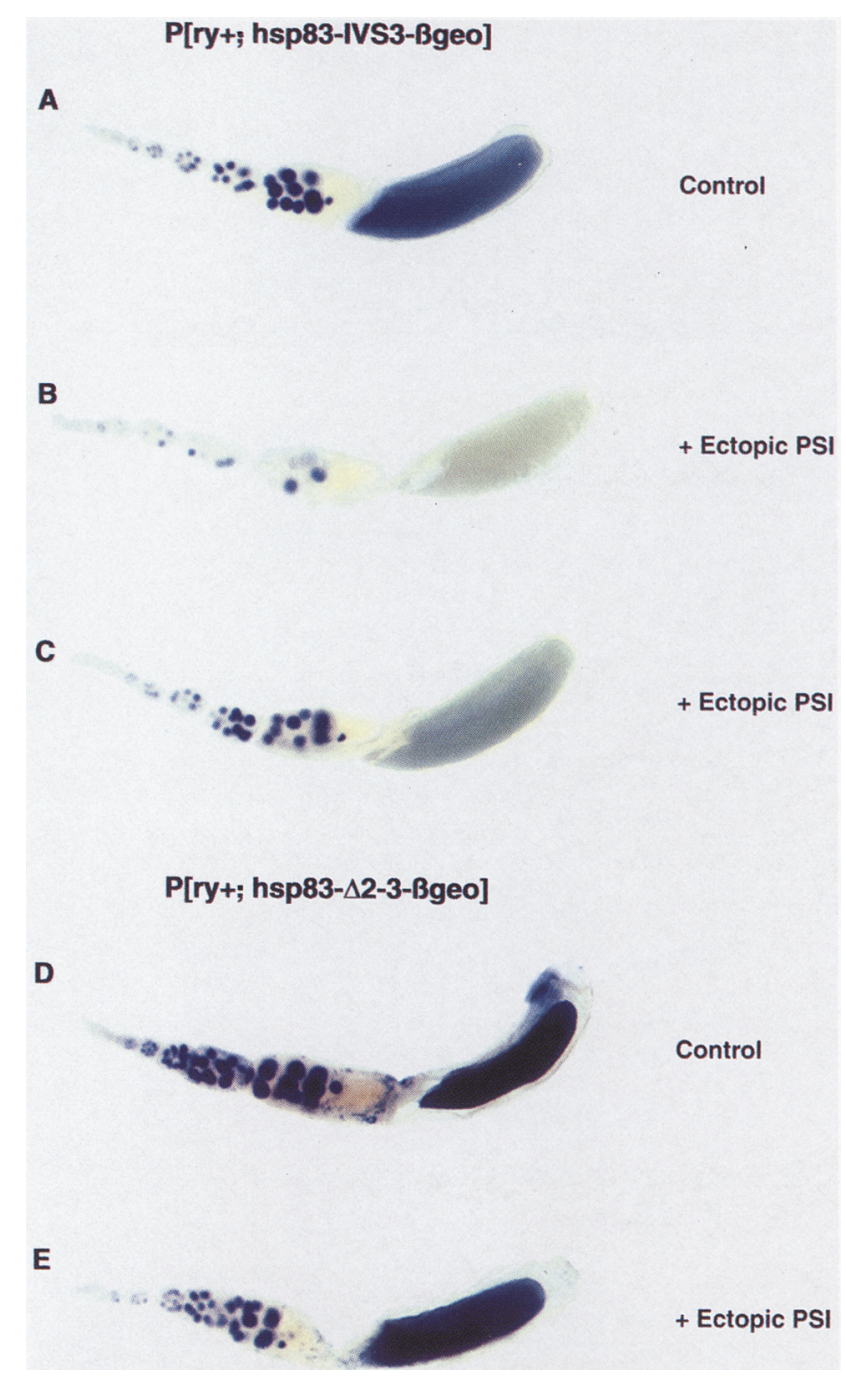

Figure 2. Ectopic expression of PSI in the female germ line inhibits splicing of an IVS3-containing reporter. $(A-C) \beta$-galactosidase staining of ovaries from female progeny containing the $\mathrm{P}\left[\mathrm{ry}^{+}{ }_{i}\right.$ hsp83-IVS3- $\beta$ geo] reporter transgene. $(A)$ Ovariole from a control (P[ry ${ }^{+}$; hsp83-IVS3-ßgeo]; CyO) female. Staining occurs in the germ-line ovarian cells throughout oogenesis. $(B, C)$ Ovarioles from two experimental ( $\mathrm{P}\left[\mathrm{ry}^{+}\right.$; hsp83-IVS3- $\beta$ geo $]$; $\mathrm{P}\left[\mathrm{w}^{+}\right.$; hsp83-PSI 29-1]) siblings. Germ-line $\beta$-galactosidase staining is repressed in the presence of ectopic PSI. The magnitude of repression varies between ovaries from independent experimental females. In the most highly repressed ovarioles $(C)$ there is a mosaic pattern of staining inhibition. $(D, E)$ Stained ovarioles from female progeny containing the $\mathrm{P}\left[\mathrm{ry}^{+}\right.$; hsp83- $\Delta 2-3-\beta$ geo $]$ reporter transgene. $(D)$ Ovariole from a control $\left(\mathrm{P}\left[\mathrm{ry}^{+} ;\right.\right.$hsp83- $\Delta 2-$ 3- $\beta$ geo]; CyO female. (E) Ovariole from an experimental (P[ry+ ${ }^{+}$hsp83- $\left.\Delta 2-3-\beta g e o\right] ; ~ P\left[w^{+}\right.$; hsp83-PSI 29-1]) female. Germline $\beta$-galactosidase staining from the $\Delta 2-3$ reporter is not affected by ectopic PSI. staining in the somatic follicle cells is evident when IVS3 is not present on the reporter.

ple (Amrein et al. 1994). PhosphorImager quantitation (see Materials and Methods) of the protected species indicated that the ratio of spliced to unspliced IVS3 RNA was reduced from 1.7 in the control to 0.6 in the experi- 
A

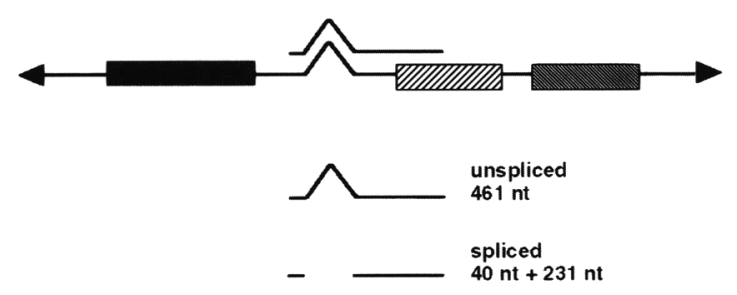

B

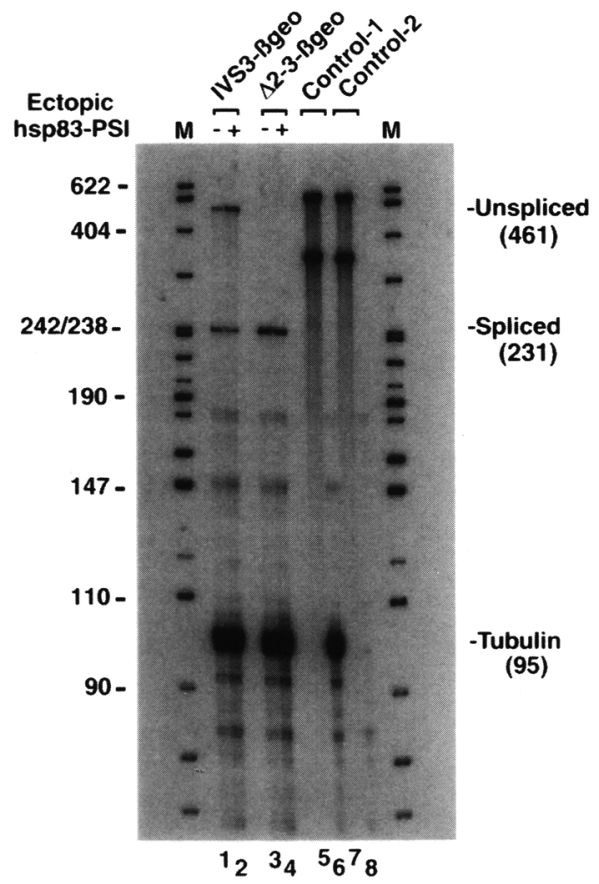

RNase Protection
C

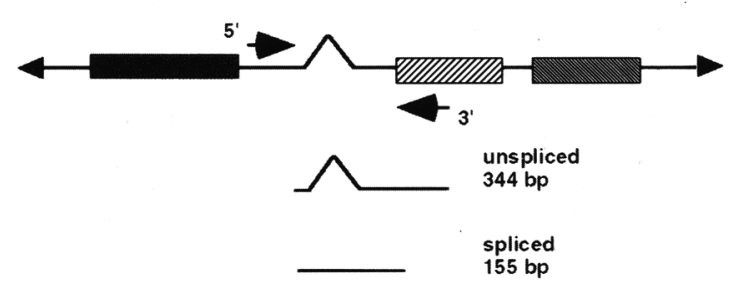

D

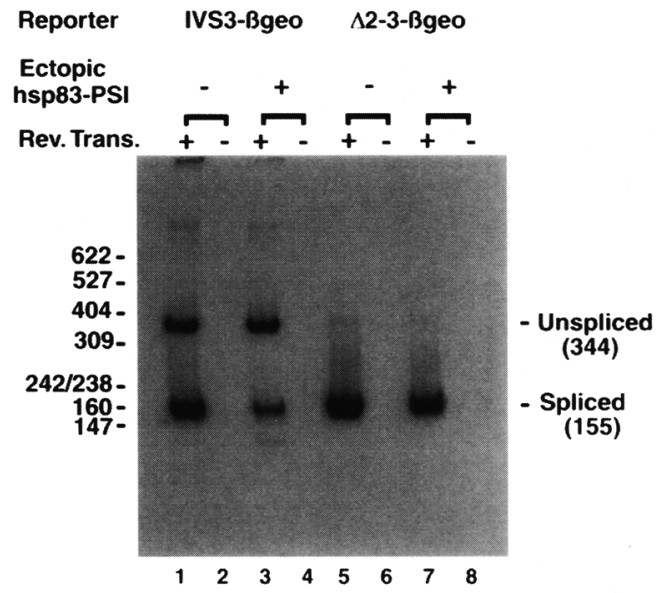

RT-PCR

Figure 3. Ectopic overexpression of PSI affects IVS3 splicing. (A) Schematic diagram of the antisense probe used for RNase protection and the probe fragments protected by unspliced and spliced IVS3-- $\beta$ geo reporter RNA. $(B)$ RNase protection analysis of total ovary RNA

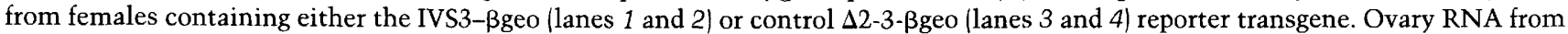
control $\left(\mathrm{P}\left[\mathrm{ry}^{+}\right.\right.$; hsp83-IVS3(or $\left.\Delta 2-3\right)-\beta$ geo]; $\mathrm{CyO}$ ) females is shown in lanes 1 and 3 and RNA from experimental (P[ry ${ }^{+}$; hsp83-IVS3/or $\Delta 2-3)$-Bgeo]; $\mathrm{P}\left[\mathrm{w}^{+}\right.$; hsp83-PSI 29-1]) females is shown in lanes 2 and 4 . The size (nt) and identity of each protected probe fragment is indicated to the right of the gel. The fragment protected by the $\Delta 2-3$ - $\beta$ geo RNA comigrates with the 231 -nucleotide probe fragement

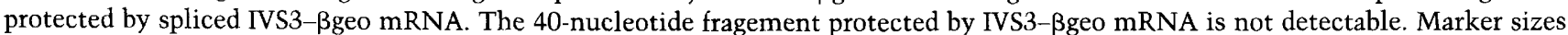
(bp) of ${ }^{32} \mathrm{P}$-labeled pBR322 cleaved with Msp I are given to the left of the gel. Values from PhosohorImager quantitiation of the products are reported in Materials and Methods. Lanes 5 and 6 contain control $\mathrm{w}^{111}$ target RNA minus (lane 5) and plus (lane 6) RNase treatment, and lanes 7 and 8 contain control total yeast RNA minus (lane 7) and plus (lane 8) RNase treatment. These controls indicate the sizes of the full-length IVS3- $\beta$ geo and tubulin probes (lanes 5 and 7) and also the protection products that are not specific for the $\beta$ geo reporter RNAs (lanes 6 and 8). (C) Schematic diagram of the oligonucleotide primers (Roche et al. 1995) used for RT-PCR and the amplification products that represent unspliced and spliced IVS3- geo reporter RNA. The 3' oligonucleotide was used to prime reverse transcription and also to amplify the resulting cDNA. (D) Quantitative RT-PCR analysis of total ovary RNA from females containing

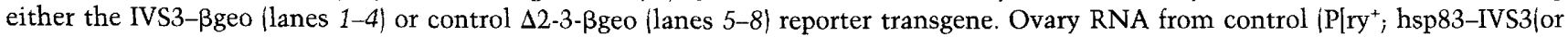

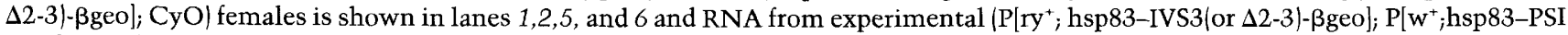
29-1]) females is shown in lanes 3,4,7, and 8 . Lanes 2,4,6, and 8 are control reactions performed in the absence of reverse transcriptase. The size (bp) and identity of each amplified cDNA is indicated to the right of the gel. Marker sizes (bp) of ${ }^{32} \mathrm{P}-\mathrm{labeled} \mathrm{pBR} 322 \mathrm{cleaved}$ with MspI are given to the left. Values from PhosphorImager quantitiation of the products are reported in Materials and Methods. 
mental ovaries, a 2.8 -fold reduction (Fig. 3B, lanes 1 and 2 ). It has been observed previously that the IVS3- $\beta$ geo reporter, as well as IVS3 from natural P elements, is incompletely spliced in the female germ line (Roche et al. 1995). The 2.8-fold reduction of the mRNA to premRNA ratio resulted from a decrease (1.7-fold) in the level of spliced IVS3 reporter RNA and a corresponding increase (1.6-fold) in the level of unspliced RNA in the presence of the PSI transgene. The total level of reporter RNA was the same in both the control and experimental samples. This quantitation confirmed that the inhibition of ovary $\beta$-galactosidase expression observed in the staining assay resulted from a decrease in germ-line IVS3 splicing in the presence of ectopic PSI.

In this particular RNase protection experiment, in the presence of ectopic PSI, there was a 2.3-fold decrease in the level of the 231-nucleotide probe fragment protected by the $\Delta 2-3$ reporter RNA relative to the control (Fig. 3B, lanes 3 and 4). However, a reduction of this magnitude for the control $\triangle 2-3$ RNA was not seen by RT-PCR on the same RNA samples (Fig. 3D, lanes 5 and 7) or in RNase protection experiments using other independent RNA preparations (data not shown). The exonic regulatory sequence on which the splicing inhibitory complex assembles in vitro is present in the control $\Delta 2-3$ reporter RNA. Thus, it is possible that binding of ectopically expressed PSI decreases the stability of this intron-less RNA in the absence of spliceosome assembly. However, we do not believe that such an effect is responsible for the reduced level of spliced RNA observed with the IVS3-containing reporter as we also see a concomitant increase in the level of unspliced RNA in the presence of the PSI transgene.

Quantitative PCR reactions were performed on reverse-transcribed total ovary RNA from the same control and experimental females using primers that flank IVS3 in the reporter transgene. cDNAs of 155 and 344 base pairs were amplified, which correspond to spliced and unspliced transcripts from the reporter transgene (Fig. 3C) (Roche et al. 1995). The relative levels of the two products in each sample were quantitated isee Materials and Methods). Consistent with the RNase protection data, the presence of ectopic PSI resulted in a threefold decrease in the ratio of spliced to unspliced IVS3 reporter RNA (Fig. 3D, lanes 1 and 3). Again, this shift in splicing was a result of both a decrease in the level of spliced mRNA and a concomitant increase in the level of unspliced reporter pre-mRNA. Analysis of ovary RNA from line 103-1 revealed a twofold reduction of the ratio of spliced to unspliced IVS3 RNA in the presence of ectopic PSI (data not shown). Taken together, these results indicate that ectopic germ-line expression of the PSI cDNA is sufficient to repress IVS3 splicing in the ovaries of transgenic females.

\section{A PSI-targeted ribozyme activates IVS3 splicing in the soma}

To date, mutations in the gene encoding PSI have not been isolated. To assess the effect of reduced PSI activity on IVS3 splicing in the soma, we generated transgenic strains of flies that express a heat-inducible hammerhead ribozyme targeted against the PSI mRNA (Fig. 4A). The PSI-specific ribozyme is under the transcriptional control of the hsp70 promoter, which is known to be active in somatic cells (Lis et al. 1983). A similar experimental approach has been used successfully to phenocopy the effect of mutations in the fushi tarazu (ftz) gene in Drosophila embryos (Zhao and Pick 1993). We also generated a reporter strain, similar to the $\mathrm{P}\left[\mathrm{ry}^{+}\right.$; hsp83-IVS3Bgeo] line described above, in which the cognate hsp70 promoter controls expression of the IVS3-IacZ fusion gene. $\beta$-Galactosidase expression from the reporter transgene requires accurate splicing of IVS3.

To determine whether a reduction in the level of PSI in somatic cells affects P-element IVS3 splicing, we crossed virgin females homozygous for the $\mathrm{P}\left[\mathrm{w}^{+} ;\right.$hsp70-PSI ribozyme] transgene to males that were homozygous for the $\mathrm{P}\left[\mathrm{w}^{+}\right.$; hsp70-IVS3-Bgeo] reporter (Fig. 4B). IVS3 splicing was assayed in early embryos because they are composed primarily of somatic cells and because high levels of PSI protein are not maternally deposited (Siebel et al. 1995; J. Warsowe and D.C. Rio, unpubl.). PSI mRNA depletion might therefore have a significant effect on the level of PSI protein. Embryos heterozygous for both transgenes were collected from the cross, aged, and subjected to a heat shock at $37^{\circ} \mathrm{C}$ to induce expression of both the PSI ribozyme and the reporter. The embryos were then assayed for $\beta$-galactosidase activity. Control embryos, heterozygous for the reporter but lacking the ribozyme transgene, were collected from a second cross and processed in parallel.

Embryos from a single transgenic line expressing the PSI-specific ribozyme showed an increase in the level of $\beta$-galactosidase activity relative to that in the control embryos (Fig. 5, cf. panels B and D). This increased staining in the presence of the PSI ribozyme is reproducible and indicates a derepression of IVS3 splicing. The staining observed following heat induction in the control embryos could be attributable to a high level of reporter RNA titrating out components of the inhibitory activity that are present in limiting amounts. A similar effect on IVS3 splicing has also been observed in Drosophila somatic tissue culture cells expressing a high level of an IVS3-ADH reporter RNA from the actin promoter (R. Eagles and D.C. Rio, unpubl.). In the absence of heat shock, staining was not observed in either control or experimental embryos (Fig. 5A,C).

RT-PCR analysis of IVS3 reporter RNA from the heatshocked embryos indicated that in the presence of the PSI-specific ribozyme, there was consistently a modest (1.2-fold) increase in the ratio of spliced to unspliced IVS3- $\beta$ geo reporter RNA (data not shown). This activation of IVS3 splicing in somatic cells appears to be sufficient to cause the observed increase in somatic $\beta$-galactosidase staining (Fig. 5). The total level of reporter RNA was similar between control and experimental samples, confirming that the activation of staining observed in the experimental embryos was not a result of transcriptional activation or a distinct process unrelated 
Figure 4. PSI ribozyme transgene and genetic assay for IVS3 splicing. (A) Schematic diagram of the transgene used to express an antisense ribozyme targeted to the PSI mRNA. Expression of the ribozyme RNA is controlled by the heat-inducible hsp 70 promoter. The sequence of the ribozyme is shown below with the most highly conserved residues in bold. The sequence of the PSI mRNA (residues $740-760$ in the cDNA sequence. Siebel et al. 1995) to which the ribozyme is complementary is also shown with the predicted cleavage site (nucleotide 750 ) indicated. (B) The genetic cross used to determine the effect of PSItargeted ribozyme on somatic IVS3 splicing. Males homozygous for the $\mathrm{P}\left[\mathrm{w}^{+}\right.$; hsp70-IVS3- $\beta$ geol reporter transgene were crossed to virgin females homozygous for the $\mathrm{P}\left[\mathrm{w}^{+}\right.$; hsp70-PSI ribozyme] transgene. Embryos (0- to 1-hr) heterozygous for each transgene were collected and aged for $1.5 \mathrm{hr}$ at $25^{\circ} \mathrm{C}$. The embryos were then incubated at either $25^{\circ} \mathrm{C}$ or heat shocked at $37^{\circ} \mathrm{C}$ for 1 $\mathrm{hr}$ before assaying for IVS3- $\beta$ geo splicing.

\section{A}

Transgene for Ribozyme Expression
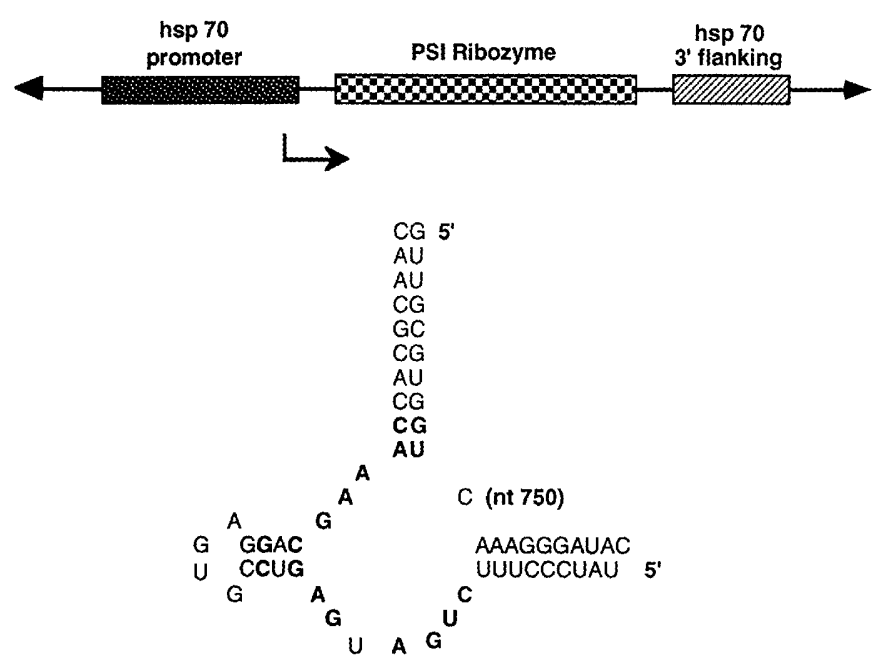

B

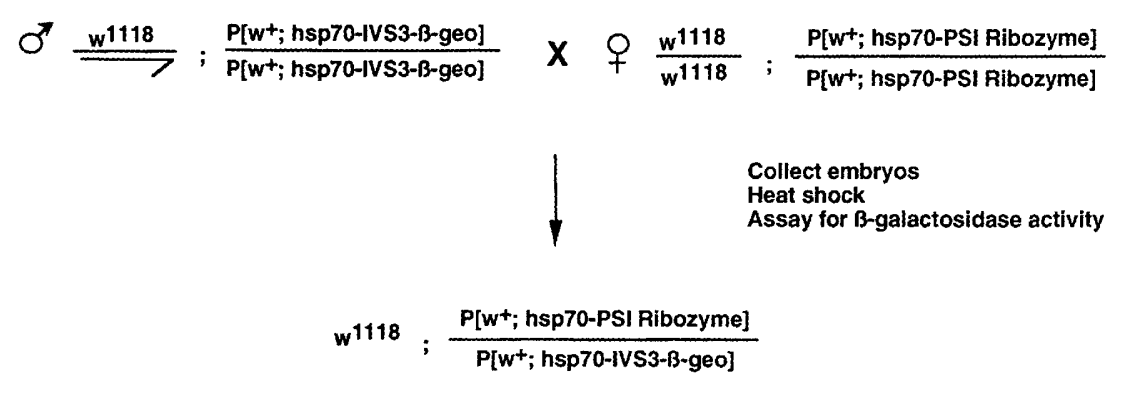

to IVS3 splicing. The magnitude of splicing activation by the PSI ribozyme may be limited by the stability of the PSI protein or by the efficiency of cleavage of PSI mRNA by the ribozyme in vivo. A dramatic decrease in the PSI protein level in the experimental embryos was not detected by whole-mount immunostaining (data not shown). Nonetheless, our data suggest that a minor reduction of PSI in somatic cells is sufficient to activate IVS3 splicing. These data further support the model that in somatic cells, PSI functions to repress P-element IVS3 splicing.

\section{Discussion}

Previous studies have shown that the PSI RNA-binding protein is required for the inhibition of IVS3 splicing observed in Drosophila somatic cell nuclear extracts. In these studies, mutations in the $5^{\prime}$ exon regulatory sequence that abolish PSI binding, as well as antibodies specific for the PSI protein, were shown to relieve inhibition and allow IVS3 to be accurately spliced in vitro (Siebel et al. 1994, 1995). Addition of recombinant PSI protein to the antibody-treated extracts restored splicing repression, showing directly that the somatic inhibitory activity requires PSI (Siebel et al. 1995). The results pre- sented here provide genetic data in support of these biochemical studies and demonstrate that PSI is a tissuespecific regulator of P-element IVS3 splicing in vivo. Here we have shown that ectopically expressed PSI represses splicing of an IVS3-lacZ fusion transcript in germ-line ovarian cells. We have also demonstrated that splicing of a similar reporter is activated in somatic embryonic cells in the presence of an antisense ribozyme specific for the PSI mRNA. These results are consistent with the hypothesis that PSI is a soma-specific splicing repressor and that the reduced level of PSI in the germ line is sufficent to allow P-element IVS3 splicing and transposase expression.

A number of observations suggest that the level of PSI protein in either the germ line or soma, or both, is tightly regulated. A high proportion of the $\mathrm{P}\left[\mathrm{w}^{+} ;\right.$hsp83-PSI $]$ transformants $(6$ of 8$)$ exhibited reduced viability when heterozygous for the transgene and could not be made into homozygous stocks. The isolation of this high percentage of P-element insertions that resulted in reduced viability implies that the deleterious biological effects observed are unlikely attributable to P-element insertions into essential loci. Because the hsp 83 promoter is active in the soma as well as the germ line, the effect of the transgenes on viability may be a result of ectopic PSI 

Control B

\section{+ PSI Ribozyme}

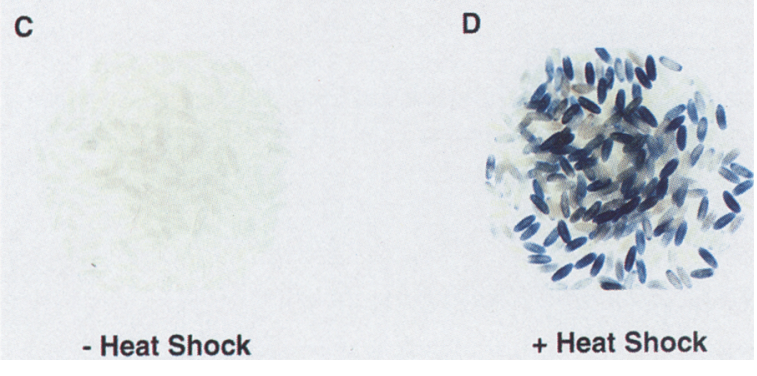

Figure 5. A PSI-targeted ribozyme activates IVS3 splicing. $(A, B)$ Control embryos that are heterozygous for the $\mathrm{P}\left[\mathrm{w}^{+}\right.$; hsp70-IVS3- $\beta$ geo $]$ reporter transgene but lack the PSI ribozyme transgene. $(C, D)$ Experimental embryos that are heterozygous for both the reporter and the $\mathrm{P}\left[\mathrm{w}^{+}\right.$; hsp70-PSI-Ribozyme $]$ transgene. Embryos in $B$ and $D$ were incubated at $37^{\circ} \mathrm{C}$ to induce the hsp 70 promoter. Somatic $\beta$-galactosidase staining is activated in the presence of the PSI-targeted ribozyme following heat induction.

expression in either tissue. The observation that neither of the healthy $\mathrm{P}\left[\mathrm{w}^{+} ;\right.$hsp83-PSI $]$transformants express a high level of ectopic PSI in the ovary is consistent with the idea that overexpression of PSI in the other transformants was responsible for their reduced viability. It has also been observed that ectopic overexpression of the Drosophila splicing factor B52/SRp55 is highly detrimental (Kraus and Lis 1994). The finding that both of the transformants that repress germ-line IVS3 splicing express only a low level of ectopic PSI in the ovary also indicates that slight variations in the level of germ-line PSI can have a significant effect on the level of IVS3 splicing. The natural P-element promoter is weak and the level of transposase mRNA in the germ line is low (Karess and Rubin 1984; Roche et al. 1995). Thus, a subtle shift in splicing of IVS3 might have a significant effect on the relative levels of P-element encoded transposase and repressor proteins in the germ line.

Our inability to demonstrate a complete repression of germ-line IVS3 splicing may result from the inability to recover and maintain transformants that express high levels of PSI in the ovary. In addition, the timing of PSI expression from the transgene may also be critical for maximal splicing repression and could be influenced by the genomic insertion position. It is also possible that factor(s) required for inhibition of IVS3 splicing, in addition to PSI, could be present only in limiting amounts in the germ line. However, an incomplete shift may not be surprising in light of the observation that when the splic- ing regulator ASF/SF2 was transiently overexpressed in mammalian cells, it induced only a partial shift in alternative splice site usage in the adenovirus E1a (Wang and Manley 1995), human $\beta$-globin, and rat $\beta$-tropomyosin (Cáceres et al. 1994) pre-mRNAs.

The finding that $\beta$-galactosidase staining, an indirect measure of IVS3 splicing, was activated in somatic embryonic cells in the presence of a PSI-specific ribozyme indicates that PSI is a component of the somatic inhibitory activity in vivo. The level of activation achieved using this approach was modest and may be limited by the activity of the ribozyme in vivo, the timing and level of ribozyme expression, the accessibility of the PSI mRNA, or by the turnover rate of the PSI protein. Isolation of mutations in the psi gene will facilitate further examination of somatic IVS3 splicing in vivo. The strong activation of $\beta$-galactosidase staining that results from only a moderate shift in splicing highlights the sensitivity of the $\beta g e o$ fusion gene as a reporter for IVS3 splicing.

Although maximal $\beta$-galactosidase staining and IVS3 splicing was observed in the presence of both the ribozyme transgene and heat induction, we did observe some staining in control embryos that contained only the $\mathrm{P}\left[\mathrm{w}^{+}\right.$; hsp70-IVS3- $\beta$ geo $]$ reporter transgene following a heat shock (Fig. 5B). We could also detect spliced reporter transcripts in total RNA from these embryos (data not shown). Using a similar staining assay, somatic IVS3 splicing was also detected in embryos expressing a reporter transgene in which the entire P-element coding sequence was fused in frame to the $l a c Z$ gene and placed under transcriptional control of the hsp70 promoter (Kitamura et al. 1993). One explanation for the somatic IVS3 splicing observed in these studies is that high levels of the reporter transcripts expressed from the hsp70 promoter following heat induction can titrate out negative regulatory factors such as PSI and limit efficient formation of the splicing inhibitory complex. A similar effect has been seen in transfection assays using an IVS3-ADH splicing reporter transcribed from the strong actin 5C promoter (R. Eagles and D.C. Rio, unpubl.). In contrast, when the P-element coding sequence alone was placed under control of the hsp70 promoter, no somatic transposase activity could be detected in the transgenic adults (Laski et al. 1986). The reason for these conflicting results is unclear. These observations could reflect differential stabilities or conformations of the $P$ element and P-element-lac $Z$ fusion transcripts that in turn could affect their abilities to bind and titrate away splicing inhibitory factors when expressed at high levels. There might also be some fundamental difference between early embryonic and adult somatic cells in their abilities to inhibit IVS3 splicing. Splicing of the $\mathrm{P}\left[\mathrm{w}^{+}\right.$; hsp70IVS3- $\beta$ geo] reporter used in this study is almost completely repressed in adult flies (L. Hammond D. Rudner, R. Kanaar, and D. Rio, in prep.). Additionally, the $\beta$-galactosidase staining assay may be more sensitive than the genetic assays for transposase activity used in the previous studies. Nevertheless, our studies indicate that perturbation of PSI expression can alter IVS3 splicing in vivo. 
The genetic data presented here support the model that PSI is a tissue-specific regulator of P-element IVS3 splicing. PSI may be the sole soma-specific alternative splicing factor that modulates the activities of general splicing factors assembled on the $5^{\prime}$ exon regulatory element in order to prevent formation of a functional spliceosome. These general factors include U1 snRNP, hrp48, and two unidentified proteins that bind to the $5^{\prime}$ exon in somatic nuclear extracts (Siebel et al. 1992). Biochemical reconstitution studies will provide further insight into the mechanistic roles of PSI, hrp48, and U1 snRNP in somatic inhibition of IVS3 splicing as well as $5^{\prime}$ splice site selection in general. The generation of mutations in the psi gene will permit further investigation of the role PSI plays in somatic inhibition in vivo and may facilitate the identification of Drosophila genes whose expression is regulated by PSI.

\section{Materials and methods}

\section{Recombinant DNA}

The $\mathrm{P}\left[\mathrm{w}^{+}\right.$; hsp83-PSI] transformation construct was generated by ligating the following four restriction fragments: the 9-kb pw8 vector (Klemenz et al. 1987) cleaved with XhoI and NotI; a 1-kb XhoI-HindIII hsp83 promoter fragment that had been amplified from plasmid pCAT82 (Gavis and Lehmann 1992) with the primers 5'-GGCGGTCGACTAACGGGAACTTGAAGAAGTG-3' and 5'-GGCGAAGCTTTATGTATGTTTTTCGTTCTATTC- ${ }^{\prime}$ and inserted into pBSIIKS $^{+}$as a SalI-HindIII fragment; a 1.8-kb HindIII-BamHI fragment from plasmid pNB40-M1 (Siebel et al. 1995) containing the PSI coding sequence; and a 1.8-kb BamHI-NotI fragment from plasmid pHSS/ $x \Delta t$ (Misra and Rio 1990) containing the SV40 small $t$ intron and polyadenylation signal. The $\mathrm{P}\left[\mathrm{w}^{+}\right.$; hsp 70 -PSI ribozyme] transformation construct was generated by ligating the pCaSpeR-hs vector cleaved with $B g l I I$ and $\mathrm{XbaI}$ to the following annealed oligonucleotides: $5^{\prime}$-GATCTGTATCCCTTTCTGATGAGTCCGTGAGGACGAAACCACGCAACT - 3 ' and 5' -CTAGAGTTGCGTGGTTTCGTCCTCACGGACTCATCAGAAAGGGATACA-3'. The $\mathrm{P}\left[\mathrm{w}^{+}\right.$; hsp70-IVS3- 3 geo $]$ transformation vector was generated by ligating the following four restriction fragments: the $9 . \mathrm{kb}$ pw8 vector cleaved with XhoI and EcoRI; a 0.4-kb fragment from pUChs (Pirrotta et al. 1985) that was cleaved with HindIII, treated with Klenow DNA polymerase I, and cleaved with EcoRI; a 1.6-kb SmaI-ClaI fragment from the plasmid pHSX-hsp83-IVS3-ßgeo (Roche et al. 1995); and a 3.4$\mathrm{kb} C l a \mathrm{I}-X h \mathrm{I}$ fragment from pPGK- $\beta$ geobpA /Friedrich and Soriano 1991).

The plasmid used to generate the antisense IVS3-lacZ probe for RNase protection was constructed from $\mathrm{pBSKS}^{+}$, which had been cleaved with PstI and SmaI, and a Klenow-treated 0.4-kb PstI-DdeI fragment from the plasmid pHSX-hsp83-IVS3- $3 g$ eo (Roche et al. 1995). The plasmid was cleaved with PstI, and T7 RNA polymerase was used for run-off transcription as described (Siebel and Rio 1990). The plasmid used to generate the tubulin probe contained the $0.4-\mathrm{kb} X b a \mathrm{I}-H$ indIII fragment of $D$. melanogaster $\alpha$-tubulin genomic sequence in pSP72 (Amrein et al. 1994). This template was cleaved with AccI and transcribed with T7 RNA polymerase.

\section{Drosophila strains and germ-line transformation}

The $\mathrm{P}\left[\mathrm{ry}^{+}\right.$; hsp83-IVS3- $\beta$ geo $]$ and $\mathrm{P}\left[\mathrm{ry}^{+}\right.$; hsp83- $\Delta 2-3-\beta$ geo] lines have been described (Roche et al. 1995). Germ-line transforma- tion of Drosophila strain $\mathrm{w}^{1118}$ was carried out using standard microinjection methods with bleach dechorionation (Spradling 1986). Isolines were established from individual independent transformants.

\section{Histochemical B-galactosidase staining assays}

Hand dissected ovaries from 3- to 4-day-old, well-fed virgin females were stained as described (O'Kane and Gehring 1987). Individual ovarioles were mounted in $90 \%$ glycerol, $50 \mathrm{~mm}$ Tris- $\mathrm{HCl}$ at $\mathrm{pH} 7.5,10 \mathrm{mg} / \mathrm{ml} \mathrm{N}$-propyl galleate. Embryos $(2.5$ to 3.5 -hour) were placed at either $25^{\circ} \mathrm{C}$ or $37^{\circ} \mathrm{C}$ for $1 \mathrm{hr}$ and then stained as described (Grossniklaus et al. 1989). Each of the staining assays was performed at least three times with similar results.

\section{RNA isolation and RNase protection analysis}

Total ovary and embryo RNA was isolated by extraction with guanidinium thiocyanate followed by high-speed centrifugation in a $\mathrm{CsCl}$ step gradient (Ausubel et al. 1987; Sambrook et al. 1989|. For RNase protection, 20-25 $\mu$ g total RNA and 50,000 cpm of each probe were coprecipitated in ethanol and resuspended in $10 \mu \mathrm{l}$ hybridization buffer $(80 \%$ deionized formamide, $0.4 \mathrm{M} \mathrm{NaCl}, 40 \mathrm{~mm}$ PIPES at $\mathrm{pH} 6.4,1 \mathrm{~mm}$ EDTA). Samples were heated for $3 \mathrm{~min}$ at $85^{\circ} \mathrm{C}$ and allowed to cool to $45^{\circ} \mathrm{C}$ over $12-16$ hr. Then $300 \mu \mathrm{l}$ of RNase A/T1 solution $10.3 \mathrm{M} \mathrm{NaCl}, 10 \mathrm{~mm}$ Tris- $\mathrm{HCl}$ at $\mathrm{pH} 7.5,5 \mathrm{~mm}$ EDTA, $400 \mathrm{U} / \mathrm{ml}$ RNase T1, $8 \mathrm{mg} / \mathrm{ml}$ RNase A) was added to each sample and digestion as carried out for $1 \mathrm{hr}$ at room temperature. Each sample was treated with 20 $\mu \mathrm{l} 10 \%$ SDS and $10 \mu 110 \mathrm{mg} / \mathrm{ml}$ proteinase $\mathrm{K}$ for $30 \mathrm{~min}$ at room temperature followed by $30 \mathrm{~min}$ at $37^{\circ} \mathrm{C}$. Samples were extracted once with phenol-chloroform and precipitated with ethanol in the presence of $2 \mu \mathrm{g}$ total yeast RNA. The RNA was resuspend in $8 \mathrm{M}$ urea plus dyes and run on a $7 \%$ denaturing polyacrylamide gel. Protected fragments were quantitated using a Molecular Dynamics Phosphorlmager and corrected for loading by comparison with the tubulin control. The corrected values were used to calculate the ratio of spliced to unspliced IVS3 RNA within each sample lane. These data are provided in the following table of PhosporImager values from RNase protection (Fig. 3B):

\begin{tabular}{lcc} 
Corrected values for & Control & $\begin{array}{l}\text { Experimental } \\
\text { (line 29-1) }\end{array}$ \\
\hline Unspliced IVS3- $\beta$ geo & 14632 & 23083 \\
Spliced IVS3- $\beta$ geo & 24832 & 14128 \\
Total reporter RNA & 39464 & 37211 \\
Ratio of spliced to unspliced & 1.7 & 0.6 \\
Control $\Delta 2-3$ reporter & 49159 & 21309 \\
\hline
\end{tabular}

RNase protection was performed on two independent RNA preparations from line 29-1.

\section{Quantitative RT-PCR analysis}

Total RNA $(50-100 \mu \mathrm{g})$ from the $\mathrm{CsCl}$ gradient was treated with $1 \mu \mathrm{g}$ DNase I (Worthington) in $10 \mathrm{~mm}$ Tris- $\mathrm{HCl}$ at $\mathrm{pH} 7.5,1 \mathrm{~mm}$ EDTA, $10 \mathrm{mM} \mathrm{MgCl}_{2}, 1 \mathrm{~mm}$ DTT, $0.04 \mathrm{U} / \mathrm{\mu l}$ RNasin (Promega) in a $100 \mu \mathrm{l} \mathrm{reaction}$ for $30 \mathrm{~min}$ at $37^{\circ} \mathrm{C}$. The RNA was extracted with phenol-chloroform and precipitated with ethanol in the 
presence of $10 \mu \mathrm{g}$ glycogen. One microgram of the DNase Itreated RNA was reverse-transcribed in a $25 \mu \mathrm{l}$ reaction using 300 units M-MLV reverse transcriptase (Gibco-BRL), 12.5 pmole of a lacZ-specific primer (Roche et al. 1995), $1.5 \mathrm{U} / \mathrm{\mu l}$ RNasin, and $1 \mathrm{~mm}$ dNTPs for $45 \mathrm{~min}$ at $37^{\circ} \mathrm{C}$. Twenty percent of the reverse transcription reaction was amplified using 12.5 pmole each of primers specific for the IVS3-lacZ gene fusion \{Roche et al. 1995) in a $25 \mu \mathrm{l}$ reaction. A time course of amplification was performed using an annealing temperature of $70^{\circ} \mathrm{C}$. Products were run on a $7 \%$ native polyacrylamide gel and electroblotted onto Hybond $\mathrm{N}^{+}$(Amersham) as described (Ausubel et al. 1987). The membrane was probed with a ${ }^{32} \mathrm{P}$-labeled $1.2-\mathrm{kb}$ XhoI-ClaI fragment from the plasmid pHSX-hsp83-IVS3-ßgeo (Roche et al. 1995). Amplified products were quantitated using a PhosphorImager and the values obtained after background subtraction for each time point were plotted semilogarithmically. Time points that fell within the linear portion of the curve were assumed to be undergoing exponential amplification and were used to calculate the ratio of spliced to unspliced IVS3 RNA within each sample lane. These data are provided in the following table of PhosphorImager values from RT-PCR (Fig. 3D):

\begin{tabular}{|c|c|c|}
\hline Corrected values for & Control & $\begin{array}{l}\text { Experimental } \\
\text { (line 29-1) }\end{array}$ \\
\hline Unspliced IVS3- $\beta$ geo & 94419 & 95448 \\
\hline Spliced IVS3- $\beta$ geo & 147620 & 48757 \\
\hline Total reporter RNA & 242039 & 144245 \\
\hline Ratio of spliced to unspliced & 1.56 & 0.51 \\
\hline Control $\Delta 2-3$ reporter & 121493 & 88186 \\
\hline
\end{tabular}

RT-PCR experiments were performed on two independent RNA preparations from line 29-1 and from embryos expressing the PSI ribozyme.

\section{Acknowledgments}

We thank Siobhan Roche for providing the hsp83-IVS3- $\beta$ geo and hsp83- $\Delta 2-3-\beta$ geo reporter constructs, as well as various reagents and protocols. We also thank $\mathrm{B}$. Tsung for technical assistance and members of the Rio laboratory for critical reading of the manuscript. This work was funded by National Institutes of Health grant R01-HD28063.

The publication costs of this article were defrayed in part by payment of page charges. This article must therefore be hereby marked "advertisement" in accordance with 18 USC section 1734 solely to indicate this fact.

\section{References}

Adams, M.D., D.Z. Rudner, and D.C. Rio. 1996. Biochemistry and regulation of pre-mRNA splicing. Curr. Opin. Cell Biol. 8: $331-339$.

Amrein, H., M.L. Hedley, and T. Maniatis. 1994. The role of specific protein-RNA and protein-protein interactions in positive and negative control of pre-mRNA splicing by Transformer 2. Cell 76: 735-746.

Ausubel, F.M., R. Brent, R.E. Kingston, D.D. Moore, J.G. Seidman, J.A. Smith, and K. Struhl. 1987. Current protocols in molecular biology. Wiley and Sons, New York, NY.

Cáceres, J.F., S. Stamm, D.M. Helfman, and A.R. Krainer. 1994. Regulation of alternative splicing in vivo by overexpression of antagonistic splicing factors. Science 265: 1706-1709.

Chain, A.C., S. Zollman, I.C. Tseng, and F.A. Laski. 1991. Identification of a cis-acting sequence required for germ linespecific splicing of the P element ORF2-ORF3 intron. Mol. Cell. Biol. 11: 1538-1546.

Ding, D., S.M. Parkhurst, S.R. Halsell, and H.D. Lipshitz. 1993. Dynamic Hsp83 RNA localization during Drosophila oogenesis and embryogenesis. Mol. Cell. Biol. 13: 3773-3781.

Friedrich, G. and P. Soriano. 1991. Promoter traps in embryonic stem cells: A genetic screen to identify and mutate developmental genes in mice. Genes \& Dev. 5: 1513-1523.

Gavis, E.R. and R. Lehmann. 1992. Localization of nanos RNA controls embryonic polarity. Cell 71: $301-313$.

Green, M.R. 1991. Biochemical mechanisms of constitutive and regulated pre-mRNA splicing. Annu. Rev. Cell Biol. 7: 559599.

Grossniklaus, U., H.J. Bellen, and W.J. Gehring. 1989. Pelement-mediated enhancer detection applied to the study of oogenesis in Drosophila. Development 107: 189-200.

Hazelrigg, T., R. Levis, and G.M. Rubin. 1984. Transformation of white locus DNA in Drosophila: Dosage compensation, zeste interaction, and position effects. Cell 36: 469-481.

Karess, R.E., and G.M. Rubin. 1984. Analysis of P transposable element functions in Drosophila. Cell 38: 135-146.

Kitamura, T., S. Kobayashi, and M. Okada. 1993. Developmentally regulated splicing of the third intron of $\mathrm{P}$ element in somatic tissues in Drosophila embryos. Dev. Growth Diff. 35: $67-73$.

Klemenz, R., U. Weber, and W.J. Gehring. 1987. The white gene as a marker in a new P-element vector for gene transfer in Drosophila. Nucleic Acids Res. 15: 3947-3959.

Kraus, M.E. and J.T. Lis. 1994. The concentration of B52, an essential splicing factor and regulator of splice site choice in vitro, is critical for Drosophila development. Mol. Cell. Biol. 14: 5360-5370.

Laski, F.A. and G.M. Rubin. 1989. Analysis of cis-acting requirements for germ-line-specific splicing of the P element ORF2ORF3 intron. Genes \& Dev. 3: 720-728.

Laski, F.A., D.C. Rio, and G.M. Rubin. 1986. Tissue specificity of Drosophila $\mathrm{P}$ element transposition is regulated at the level of mRNA splicing. Cell 4: 7-19.

Lis, J.T., J.A. Simon, and C.A. Sutton. 1983. New heat shock puffs and $\beta$-galactosidase activity resulting from transformation of Drosophila with an hsp70-lacz hybrid gene. Cell 35: 403-410.

Matunis, E.L., M.J. Matunis, and G. Dreyfuss. 1992b. Characterization of the major hnRNP proteins from Drosophila melanogaster. I. Cell Biol, 116: 257-269.

. 1992a. Isolation of hnRNP complexes from Drosophila melanogaster. J. Cell Biol. 116: 245-255.

Misra, S. and D.C. Rio. 1990. Cytotype control of Drosophila P element transposition: The $66 \mathrm{kD}$ protein is a repressor of transposase activity. Cell 62: 269-284.

Moore, M.J., C.C. Query, and P.A. Sharp. 1993. Splicing of precursors to messenger RNAs by the spliceosome. In RNA world (ed. R.F. Gestland and J.F. Atkinsl, pp. 303-357. Cold Spring Harbor Laboratory Press, Cold Spring Harbor, NY.

O'Kane, C.J., and W.J. Gehring. 1987. Detection in situ of genomic regulatory elements in Drosophila. Proc. Natl. Acad. Sci. 84: 9123-9127.

Pirrotta, V., H. Steller, and M.P. Bozzetti. 1985. Multiple upstream regulatory elements control the expression of the Drosophila white gene. EMBO J. 4: 3501-3508.

Rio, D.C. 1993. Splicing of pre-mRNA: Mechanism, regulation and role in development. Curr. Opin. Genet. Dev. 3: 574584. 
Adams et al.

Roche, S.E., M. Schiff, and D.C. Rio. 1995. P-element repressor autoregulation involves germ-line trancriptional repression and reduction of third intron splicing. Genes \& Dev. 9: $1278-1288$.

Sambrook, J., E.F. Fritsch, and T. Maniatis. 1989. Molecular cloning: A laboratory manual. Cold Spring Harbor Laboratory Press, Cold Spring Harbor, NY.

Siebel, C.W., and D.C. Rio. 1990. Regulated splicing of the Drosophila $\mathrm{P}$ transposable element third intron in vitro: Somatic repression. Science 248: 1200-1208.

Siebel, C.W., L.D. Fresco, and D.C. Rio. 1992. The mechanism of somatic inhibition: Multiprotein complexes at an exon pseudo- $5^{\prime}$ splice site control U1 snRNP binding. Genes \& Dev. 6: 1386-1401.

Siebel, C.W., R. Kanaar, and D.C. Rio. 1994. Regulation of tissue-specific $\mathrm{P}$ element pre-mRNA splicing requires the RNA-binding protein PSI. Genes \& Dev. 8: 1713-1725.

Siebel, C.W., A. Admon, and D.C. Rio. 1995. Soma-specific expression and cloning of PSI, a negataive regulator of $\mathrm{P}$ element pre-mRNA splicing. Genes \& Dev. 9: 269-283.

Spradling, A.C. 1986. P element-mediated transformation. In Drosophila: A practical approach (ed. D. Roberts), pp.175197. IRL Press, Washington, D.C.

Wang, J. and J.F. Manley. 1995. Overexpression of the SR proteins ASF/SF2 and SC35 influences alternative splicing in vivo in diverse ways. RNA 1: 335-346.

Zhao, J.J. and L. Pick. 1993. Generating loss-of-function phenotypes of the fushi tarazu gene with a targeted ribozyme in Drosophila. Nature 365: 448-451. 


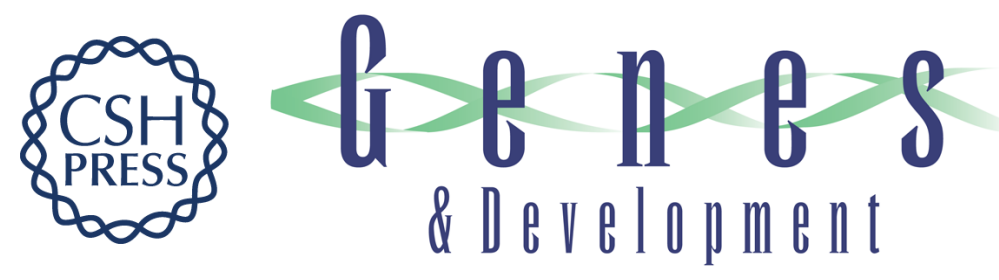

\section{The alternative splicing factor PSI regulates P-element third intron splicing in vivo.}

M D Adams, R S Tarng and D C Rio

Genes Dev. 1997, 11:

Access the most recent version at doi:10.1101/gad.11.1.129

References This article cites 30 articles, 16 of which can be accessed free at: http://genesdev.cshlp.org/content/11/1/129.full.html\#ref-list-1

License

Email Alerting

Service

Receive free email alerts when new articles cite this article - sign up in the box at the top right corner of the article or click here.

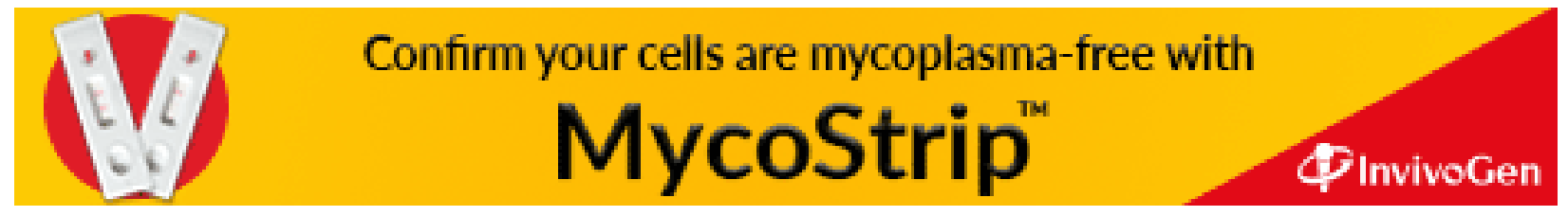

